\title{
Absence of Suppression in Particle Production at Large Transverse Momentum in $\sqrt{s_{N N}}=200 \mathrm{GeV} \mathrm{d}+\mathrm{Au}$ Collisions
}

S.S. Adler, ${ }^{4}$ S. Afanasiev, ${ }^{19}$ C. Aidala,${ }^{9}$ N.N. Ajitanand,${ }^{43}$ Y. Akiba, ${ }^{20,}{ }^{39}$ A. Al-Jamel,${ }^{34}$ J. Alexander, ${ }^{43}$ K. Aoki ${ }^{24}$ L. Aphecetche, ${ }^{45}$ R. Armendariz,${ }^{34}$ S.H. Aronson, ${ }^{4}$ R. Averbeck, ${ }^{44}$ T.C. Awes,${ }^{35}$ V. Babintsev,${ }^{16}$ A. Baldisseri, ${ }^{10}$ K.N. Barish,${ }^{5}$ P.D. Barnes,${ }^{27}$ B. Bassalleck,${ }^{33}$ S. Bathe,${ }^{50}$ S. Batsouli, ${ }^{9}$ V. Baublis,${ }^{38}$ F. Bauer,${ }^{5}$ A. Bazilevsky, ${ }^{4,40}$ S. Belikov, ${ }^{18,16}$ M.T. Bjorndal, ${ }^{9}$ J.G. Boissevain, ${ }^{27}$ H. Borel,${ }^{10}$ M.L. Brooks, ${ }^{27}$ D.S. Brown, ${ }^{34}$ N. Bruner, ${ }^{33}$ D. Bucher, ${ }^{30}$ H. Buesching, ${ }^{40}$ V. Bumazhnov, ${ }^{16}$ G. Bunce, ${ }^{4,}{ }^{40}$ J.M. Burward-Hoy, ${ }^{27,26}$ S. Butsyk, ${ }^{44}$ X. Camard, ${ }^{45}$ P. Chand,${ }^{3}$ W.C. Chang, ${ }^{2}$ S. Chernichenko, ${ }^{16}$ C.Y. Chi,${ }^{9}$ J. Chiba, ${ }^{20}$ M. Chiu, ${ }^{9}$ I.J. Choi,${ }^{52}$ R.K. Choudhury ${ }^{3}$ T. Chujo, ${ }^{4}$ V. Cianciolo, ${ }^{35}$ Y. Cobigo, ${ }^{10}$ B.A. Cole,${ }^{9}$ M.P. Comets,${ }^{36}$ P. Constantin, ${ }^{18}$ M. Csanád, ${ }^{12}$ T. Csörgő, ${ }^{21}$ J.P. Cussonneau ${ }^{45}$ D. d'Enterria,${ }^{9}$ K. Das, ${ }^{13}$ G. David,${ }^{4}$ F. Deák, ${ }^{12}$ H. Delagrange ${ }^{45}$ A. Denisov, ${ }^{16}$ A. Deshpande, ${ }^{40}$ E.J. Desmond ${ }^{4}$ A. Devismes,${ }^{44}$ O. Dietzsch,${ }^{41}$ J.L. Drachenberg, ${ }^{1}$ O. Drapier, ${ }^{25}$ A. Drees ${ }^{44}$ A. Durum, ${ }^{16}$ D. Dutta,${ }^{3}$ V. Dzhordzhadze, ${ }^{46}$ Y.V. Efremenko, ${ }^{35}$ H. En'yo, ${ }^{39,}, 40$ B. Espagnon, ${ }^{36}$ S. Esumi,${ }^{48}$ D.E. Fields, ${ }^{33,}{ }^{40}$ C. Finck,${ }^{45}$ F. Fleuret, ${ }^{25}$ S.L. Fokin, ${ }^{23}$ B.D. Fox ${ }^{40}$ Z. Fraenkel, ${ }^{51}$ J.E. Frantz, ${ }^{9}$ A. Franz,${ }^{4}$ A.D. Frawley, ${ }^{13}$ Y. Fukao,,${ }^{24,39,40}$ S.-Y. Fung, ${ }^{5}$ S. Gadrat, ${ }^{28}$ M. Germain, ${ }^{45}$ A. Glenn, ${ }^{46}$ M. Gonin, ${ }^{25}$ J. Gosset, ${ }^{10}$ Y. Goto, ${ }^{39,40}$ R. Granier de Cassagnac, ${ }^{25}$ N. Grau, ${ }^{18}$ S.V. Greene, ${ }^{49}$ M. Grosse Perdekamp,${ }^{17,40}$ H.-A. Gustafsson, ${ }^{29}$ T. Hachiya, ${ }^{15}$ J.S. Haggerty, ${ }^{4}$ H. Hamagaki, ${ }^{7}$ A.G. Hansen,${ }^{27}$ E.P. Hartouni, ${ }^{26}$ M. Harvey, ${ }^{4}$ K. Hasuko, ${ }^{39}$ R. Hayano, ${ }^{7}$ X. He,${ }^{14}$ M. Heffner,${ }^{26}$ T.K. Hemmick, ${ }^{44}$ J.M. Heuser,${ }^{39}$ P. Hidas, ${ }^{21}$ H. Hiejima, ${ }^{17}$ J.C. Hill, ${ }^{18}$ R. Hobbs, ${ }^{33}$ W. Holzmann, ${ }^{43}$ K. Homma, ${ }^{15}$ B. Hong, ${ }^{22}$ A. Hoover,${ }^{34}$ T. Horaguchi, ${ }^{39,40,47}$ T. Ichihara, ${ }^{39,40}$ V.V. Ikonnikov, ${ }^{23}$ K. Imai, ${ }^{24,39}$ M. Inuzuka, ${ }^{7}$ D. Isenhower, ${ }^{1}$ L. Isenhower, ${ }^{1}$ M. Issah, ${ }^{43}$ A. Isupov ${ }^{19}$ B.V. Jacak, ${ }^{44}$ J. Jia,${ }^{44}$ O. Jinnouchi, ${ }^{39,}{ }^{40}$ B.M. Johnson, ${ }^{4}$ S.C. Johnson, ${ }^{26}$ K.S. Joo, ${ }^{31}$ D. Jouan, ${ }^{36}$ F. Kajihara,${ }^{7}$ S. Kametani, ${ }^{7,50}$ N. Kamihara,${ }^{39,47}$ M. Kaneta, ${ }^{40}$ J.H. Kang, ${ }^{52}$ K. Katou, ${ }^{50}$ T. Kawabata, ${ }^{7}$ A. Kazantsev, ${ }^{23}$ S. Kelly, ${ }^{8,9}$ B. Khachaturov ${ }^{51}$ A. Khanzadeev, ${ }^{38}$ J. Kikuchi, ${ }^{50}$ D.J. Kim, ${ }^{52}$ E. Kim, ${ }^{42}$ G.-B. Kim,${ }^{25}$ H.J. Kim, ${ }^{52}$ E. Kinney, ${ }^{8}$ A. Kiss,${ }^{12}$ E. Kistenev, ${ }^{4}$ A. Kiyomichi, ${ }^{39}$ C. Klein-Boesing, ${ }^{30}$ H. Kobayashi, ${ }^{40}$ V. Kochetkov, ${ }^{16}$ R. Kohara, ${ }^{15}$ B. Komkov, ${ }^{38}$ M. Konno, ${ }^{48}$ D. Kotchetkov, ${ }^{5}$ A. Kozlov ${ }^{51}$ P.J. Kroon, ${ }^{4}$ C.H. Kuberg, ${ }^{1}$ G.J. Kunde,${ }^{27}$ K. Kurita, ${ }^{39}$ M.J. Kweon, ${ }^{22}$ Y. Kwon,${ }^{52}$ G.S. Kyle, ${ }^{34}$ R. Lacey, ${ }^{43}$ J.G. Lajoie, ${ }^{18}$ Y. Le Bornec, ${ }^{36}$ A. Lebedev,${ }^{18,23}$ S. Leckey, ${ }^{44}$ D.M. Lee ${ }^{27}$ M.J. Leitch, ${ }^{27}$ M.A.L. Leite ${ }^{41}$ X. Li,${ }^{6}$ X.H. Li,${ }^{5}$ H. Lim,${ }^{42}$ A. Litvinenko, ${ }^{19}$ M.X. Liu,${ }^{27}$ C.F. Maguire, ${ }^{49}$ Y.I. Makdisi, ${ }^{4}$ A. Malakhov, ${ }^{19}$ V.I. Manko, ${ }^{23}$ Y. Mao, ${ }^{37,}{ }^{39}$ G. Martinez ${ }^{45}$ H. Masui ${ }^{48}$ F. Matathias, ${ }^{44}$ T. Matsumoto, ${ }^{7,}{ }^{50}$ M.C. McCain,${ }^{1}$ P.L. McGaughey, ${ }^{27}$

Y. Miake, ${ }^{48}$ T.E. Miller ${ }^{49}$ A. Milov,${ }^{44}$ S. Mioduszewski,${ }^{4}$ G.C. Mishra, ${ }^{14}$ J.T. Mitchell, ${ }^{4}$ A.K. Mohanty,${ }^{3}$

D.P. Morrison, ${ }^{4}$ J.M. Moss,${ }^{27}$ D. Mukhopadhyay, ${ }^{51}$ M. Muniruzzaman, ${ }^{5}$ S. Nagamiya, ${ }^{20}$ J.L. Nagle,${ }^{8,9}$

T. Nakamura, ${ }^{15}$ J. Newby, ${ }^{46}$ A.S. Nyanin, ${ }^{23}$ J. Nystrand ${ }^{29}$ E. O’Brien, ${ }^{4}$ C.A. Ogilvie, ${ }^{18}$ H. Ohnishi, ${ }^{39}$ I.D. Ojha, ${ }^{49}$ H. Okada, ${ }^{24,39}$ K. Okada, ${ }^{39,} 40$ A. Oskarsson, ${ }^{29}$ I. Otterlund, ${ }^{29}$ K. Oyama,${ }^{7}$ K. Ozawa, ${ }^{7}$ D. Pal, ${ }^{51}$ A.P.T. Palounek,,${ }^{27}$

V. Pantuev, ${ }^{44}$ V. Papavassiliou, ${ }^{34}$ J. Park, ${ }^{42}$ W.J. Park ${ }^{22}$ S.F. Pate ${ }^{34}$ H. Pei,${ }^{18}$ V. Penev, ${ }^{19}$ J.-C. Peng, ${ }^{17}$

H. Pereira, ${ }^{10}$ V. Peresedov, ${ }^{19}$ A. Pierson, ${ }^{33}$ C. Pinkenburg, ${ }^{4}$ R.P. Pisani,${ }^{4}$ M.L. Purschke, ${ }^{4}$ A.K. Purwar,${ }^{44}$ J. Qualls, ${ }^{1}$ J. Rak, ${ }^{18}$ I. Ravinovich, ${ }^{51}$ K.F. Read,${ }^{35,46}$ M. Reuter, ${ }^{44}$ K. Reygers,${ }^{30}$ V. Riabov ${ }^{38}$ Y. Riabov ${ }^{38}$ G. Roche, ${ }^{28}$ A. Romana, ${ }^{25}$ M. Rosati, ${ }^{18}$ S. Rosendahl, ${ }^{29}$ P. Rosnet, ${ }^{28}$ V.L. Rykov, ${ }^{39}$ S.S. Ryu, ${ }^{52}$ N. Saito, ${ }^{24,}, 39,40$ T. Sakaguchi, ${ }^{7,50}$ S. Sakai, ${ }^{48}$ V. Samsonov, ${ }^{38}$ L. Sanfratello, ${ }^{33}$ R. Santo, ${ }^{30}$ H.D. Sato, ${ }^{24,}{ }^{39}$ S. Sato,, 48 S. Sawada, ${ }^{40}$ Y. Schutz, ${ }^{45}$ V. Semenov, ${ }^{16}$ R. Seto,${ }^{5}$ T.K. Shea,${ }^{4}$ I. Shein, ${ }^{16}$ T.-A. Shibata, ${ }^{39,}, 47$ K. Shigaki, ${ }^{15}$ M. Shimomura ${ }^{48}$ A. Sickles ${ }^{44}$ C.L. Silva, ${ }^{41}$ D. Silvermyr,${ }^{27}$ K.S. Sim, ${ }^{22}$ A. Soldatov, ${ }^{16}$ R.A. Soltz, ${ }^{26}$ W.E. Sondheim, ${ }^{27}$ S. Sorensen, ${ }^{46}$ I.V. Sourikova, ${ }^{4}$ F. Staley, ${ }^{10}$ P.W. Stankus, ${ }^{35}$ E. Stenlund,${ }^{29}$ M. Stepanov, ${ }^{34}$ A. Ster,${ }^{21}$ S.P. Stoll, ${ }^{4}$ T. Sugitate ${ }^{15}$ J.P. Sullivan, ${ }^{27}$ S. Takagi, ${ }^{48}$ E.M. Takagui, ${ }^{41}$ A. Taketani, ${ }^{39,}, 40$ Y. Tanaka, ${ }^{32}$ K. Tanida, ${ }^{39}$ M.J. Tannenbaum, ${ }^{4}$

A. Taranenko, ${ }^{43}$ P. Tarján, ${ }^{11}$ T.L. Thomas, ${ }^{33}$ M. Togawa, ${ }^{24,}{ }^{39}$ J. Tojo, ${ }^{39}$ H. Torii, ${ }^{24,40}$ R.S. Towell, ${ }^{1}$ V-N. Tram, ${ }^{25}$

I. Tserruya, ${ }^{51}$ Y. Tsuchimoto, ${ }^{15}$ H. Tydesjö, ${ }^{29}$ N. Tyurin,,${ }^{16}$ T.J. Uam,${ }^{31}$ H.W. van Hecke, ${ }^{27}$ J. Velkovska, ${ }^{4}$

M. Velkovsky, ${ }^{44}$ V. Veszprémi, ${ }^{11}$ A.A. Vinogradov ${ }^{23}$ M.A. Volkov, ${ }^{23}$ E. Vznuzdaev, ${ }^{38}$ X.R. Wang, ${ }^{14}$

Y. Watanabe, ${ }^{39,40}$ S.N. White, ${ }^{4}$ N. Willis, ${ }^{36}$ F.K. Wohn, ${ }^{18}$ C.L. Woody,${ }^{4}$ W. Xie,${ }^{5}$ A. Yanovich, ${ }^{16}$ S. Yokkaichi, ${ }^{39,} 40$ G.R. Young, ${ }^{35}$ I.E. Yushmanov, ${ }^{23}$ W.A. Zajc, ${ }^{9}$, C. Zhang, ${ }^{9}$ S. Zhou, ${ }^{6}$ J. Zimányi, ${ }^{21}$ L. Zolin, ${ }^{19}$ and X. Zong ${ }^{18}$

(PHENIX Collaboration)

\footnotetext{
${ }^{1}$ Abilene Christian University, Abilene, TX 79699, USA

${ }^{2}$ Institute of Physics, Academia Sinica, Taipei 11529, Taiwan

${ }^{3}$ Bhabha Atomic Research Centre, Bombay 400 085, India

${ }^{4}$ Brookhaven National Laboratory, Upton, NY 11973-5000, USA
} 


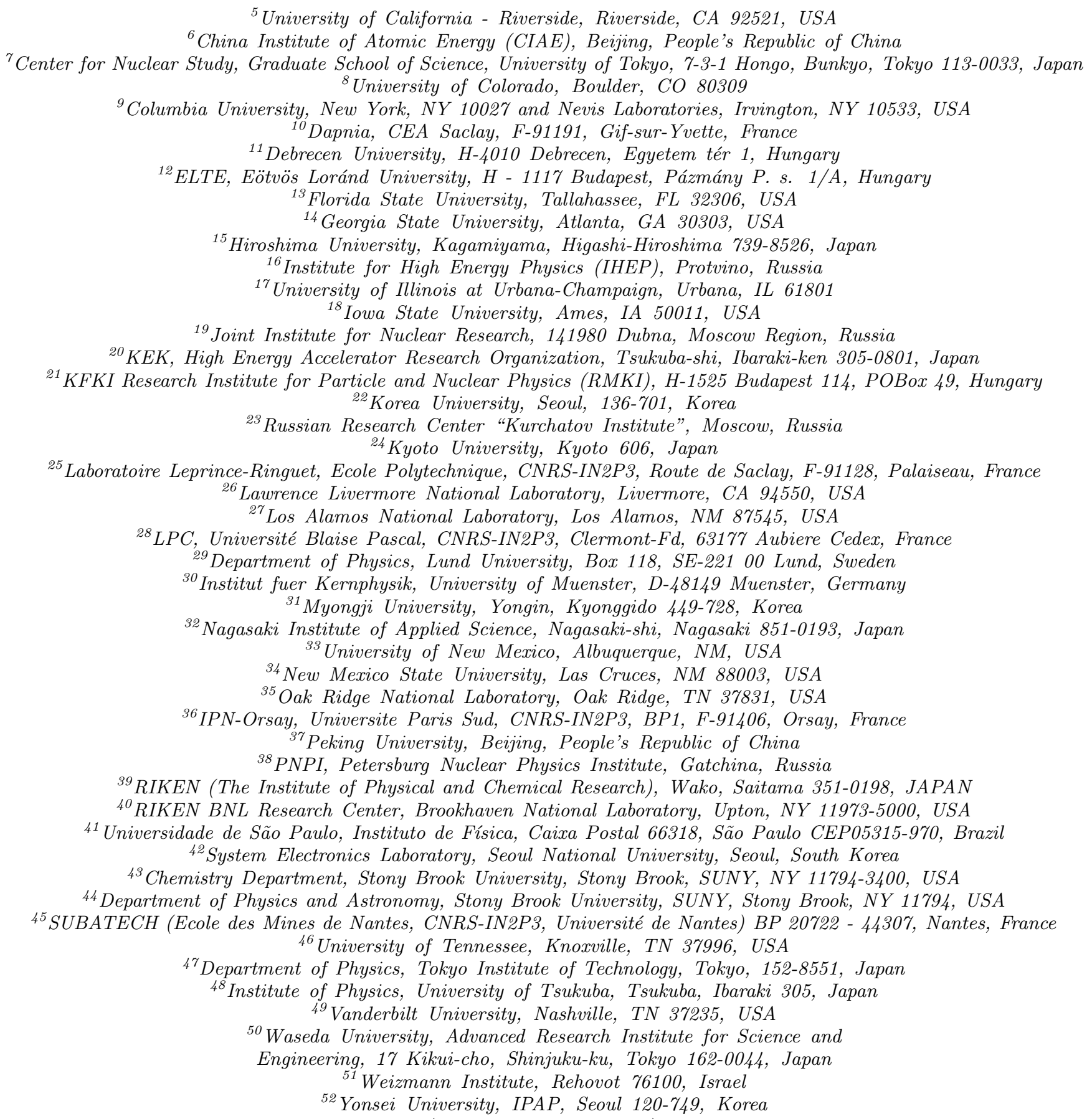

(Dated: October 25, 2018)

\begin{abstract}
Transverse momentum spectra of charged hadrons with $p_{T}<8 \mathrm{GeV} / c$ and neutral pions with $p_{T}<10 \mathrm{GeV} / c$ have been measured at midrapidity by the PHENIX experiment at RHIC in $\mathrm{d}+\mathrm{Au}$ collisions at $\sqrt{s_{N N}}=200 \mathrm{GeV}$. The measured yields are compared to those in $\mathrm{p}+\mathrm{p}$ collisions at the same $\sqrt{s_{N N}}$ scaled up by the number of underlying nucleon-nucleon collisions in $\mathrm{d}+\mathrm{Au}$. The yield ratio does not show the suppression observed in central $\mathrm{Au}+\mathrm{Au}$ collisions at RHIC. Instead, there is a small enhancement in the yield of high momentum particles.
\end{abstract}

PACS numbers: 25.75.Dw

High transverse momentum $\left(p_{T}>2 \mathrm{GeV} / c\right)$ hadrons provide an excellent probe of the high energy density matter created in relativistic heavy ion collisions [1, 2].
They arise from fragmentation of quarks and gluons (partons) scattered with large momentum transfer, $Q^{2}$, in the initial parton-parton interactions [3]. In the absence of 
medium effects, these hard scattering yields in nucleusnucleus collisions should scale with the average number of inelastic nucleon-nucleon collisions $N_{\text {coll }}$ (binary scaling). One of the most intriguing observations from experiments at the Relativistic Heavy Ion Collider (RHIC) is the large suppression of high $p_{T}$ neutral pion and charged hadron yields in central $\mathrm{Au}+\mathrm{Au}$ collisions with respect to $\mathrm{p}+\mathrm{p}$ results scaled by the number of binary nucleon-nucleon collisions 4, 5, 6, 7].

Theoretical studies of parton propagation in high density matter suggest that partons lose a significant fraction of their energy through gluon bremsstrahlung 1, 2], reducing the parton momentum and depleting the yield of high $p_{T}$ hadrons [8, 9, 10, 11, 12, 13]. This is a final-state effect in the spatially extended medium created in $\mathrm{A}+\mathrm{A}$ collisions. Initial state effects include nuclear modifications to the parton momentum distributions (structure functions), and soft scatterings of the incoming parton prior to its hard scattering. These should be present in $\mathrm{p}+\mathrm{A}, \mathrm{d}+\mathrm{A}$ and $\mathrm{A}+\mathrm{A}$. Interpretations of $\mathrm{Au}+\mathrm{Au}$ collisions based on initial-state parton saturation effects [14] or final-state hadronic interactions 15] also predict a considerable suppression of the hadron production at high $p_{T}$. It is therefore of paramount interest to determine experimentally the modification, if any, of high $p_{T}$ hadron yields due to initial state nuclear effects for a system in which a hot, dense medium is not produced in the final state. This Letter reports on charged hadron and $\pi^{0}$ production at midrapidity obtained by the PHENIX [16] experiment at RHIC in $\mathrm{d}+\mathrm{Au}$ collisions at $\sqrt{s_{N N}}=200 \mathrm{GeV}$. The results are compared to those in $\mathrm{p}+\mathrm{p}$ and $\mathrm{Au}+\mathrm{Au}$ [7, 17, 18] at the same nucleon-nucleon center-of-mass energy. Similar $\mathrm{d}+\mathrm{Au}$ measurements are also reported in [19, 20].

The data were collected under two different trigger conditions. Minimum bias events with vertex position along the beam axis within $|z|<30 \mathrm{~cm}$ were triggered by the Beam-Beam Counters (BBC) [16] which cover $|\eta|=3.0$ 3.9. The minimum bias trigger accepts $(88 \pm 4 \%)$ of all inelastic $\mathrm{d}+\mathrm{Au}$ collisions that satisfy the vertex condition. A total of $1.2 \times 10^{7}$ and $1.7 \times 10^{7}$ events were analyzed for charged hadron and $\pi^{0}$ spectra, respectively. A second "photon triggered" sample, requiring showers above $2.5 \mathrm{GeV}$ for lead-scintillator (PbSc) and $3.5 \mathrm{GeV}$ for lead-glass (PbGl) Electromagnetic Calorimeters (EMCal) [16] in addition to the $\mathrm{BBC}$ requirement, is used to extend the $\pi^{0}$ measurements to higher $p_{T}$. This trigger sampled a total of $1.7 \times 10^{9}$ events.

Neutral pions were measured by the PHENIX EMCal via the $\pi^{0} \rightarrow \gamma \gamma$ decay. The EMCal covers $|\eta| \leq 0.35$ in pseudorapidity and $\Delta \phi=\pi$ in azimuth. It consists of $6 \mathrm{PbSc}$ and $2 \mathrm{PbGl}$ sectors, each covering $\pi / 8$ in azimuth. The data from $\mathrm{PbSc}$ and $\mathrm{PbGl}$ were analyzed separately. The energy calibration is obtained from beam tests, cosmic rays, minimum ionizing energy peaks of charged hadrons, and the energy/momentum ratio of
TABLE I: Systematic errors in percent on $\pi^{0}$ invariant yields for $\mathrm{PbSc}(\mathrm{PbGl})$, as a function of $p_{T}$ (in $\left.\mathrm{GeV} / c\right)$. There are 3 categories of uncertainty: Type $\mathbf{A}$ is a point-to-point error uncorrelated between $p_{T}$ bins, type $\mathbf{B}$ is $p_{T}$ correlated, all points move in the same direction but not by the same factor, while in type $\mathbf{C}$ all points move by the same factor independent of $p_{T}$.

\begin{tabular}{ccccc}
\hline \hline & type & $p_{T}=2$ & $p_{T}=6$ & $p_{T}=10$ \\
\hline peak extraction & $\mathbf{A}$ & $5.0(5.0)$ & $5.0(5.0)$ & $5.0(5.0)$ \\
geom. accept. & $\mathbf{B}$ & $3.0(3.0)$ & $2.0(2.0)$ & $2.0(2.0)$ \\
$\pi^{0}$ reconstr.eff. & $\mathbf{B}$ & $4.0(4.0)$ & $4.0(4.0)$ & $4.5(4.5)$ \\
energy scale & $\mathbf{B}$ & $4.0(4.0)$ & $9.0(9.0)$ & $11.0(11.0)$ \\
trigger eff. & $\mathbf{B}$ & - & $5.0(10.0)$ & $3.0(3.0)$ \\
trigger norm. & $\mathbf{C}$ & - & $5.0(5.0)$ & $5.0(5.0)$ \\
conversion corr. & $\mathbf{C}$ & $2.8(2.8)$ & $2.8(2.8)$ & $2.8(2.8)$ \\
\hline total error & & $8.6(8.6)$ & $14(16)$ & $15(15)$ \\
\hline \hline
\end{tabular}

electrons identified with the Ring Imaging Čerenkov Detector $(\mathrm{RICH})$. The combined uncertainty on the energy scale and linearity is $\leq 1.5 \%$, determined from the response to identified electrons, and confirmed by the positions and widths of the observed $\pi^{0}$ mass peaks.

Photon-like energy clusters in the calorimeter are selected via shower profile cuts. The invariant mass for all photon pairs with energy asymmetry $\left|E_{1}-E_{2}\right| /\left(E_{1}+\right.$ $\left.E_{2}\right)<0.7$ is calculated and binned in $p_{T}$. The $\pi^{0}$ yield in each $p_{T}$ bin is determined by integrating the background subtracted two-photon invariant mass distribution [7]; the background is determined from mixed events. The peak-to-background ratio increases from $\sim 0.3$ at $p_{T}=1.25 \mathrm{GeV} / c$ to more than 5 above $4.25 \mathrm{GeV} / c$. The raw $\pi^{0}$ spectra are corrected by Monte Carlo simulations for trigger efficiency, acceptance, and for $\pi^{0}$ reconstruction efficiency including dead areas, effects of energy resolution, photon identification cuts and peak extraction window. Finally, the yields are corrected to the center of the $p_{T}$ bin using the observed slope of the spectrum. Below $p_{T}=5 \mathrm{GeV} / c$ the yields are determined from the minimum bias data sample while above $5 \mathrm{GeV} / c$ the photon triggered sample is used. The main sources of systematic errors are listed in Table I. The final systematic errors on the spectra are 10 to $16 \%$, increasing with $p_{T}$.

Charged particles are reconstructed using a drift chamber (DC) followed by two layers of multiwire proportional chambers with pad readout (PC1, PC3) 16]. In this analysis tracks were reconstructed over a restricted pseudorapidity range $|\eta|<0.18$. Pattern recognition in the DC is based on a combinatorial Hough transform in the track bend plane, while the polar angle is determined by PC1 and the location of the collision vertex along the beam direction 21]. The vertex was constrained to be within $|z|<18 \mathrm{~cm}$ for analysis of the charged tracks. The track reconstruction efficiency is approximately $98 \%$, in- 
TABLE II: Systematic errors in percent on the $\left(h^{+}+h^{-}\right) / 2$ invariant yields. Error types are as in Table I.

\begin{tabular}{cccccc}
\hline \hline & type & $p_{T}<4$ & $p_{T}=4$ & $p_{T}=6$ & $p_{T}=7$ \\
\hline PC3 match & $\mathbf{C}$ & 2.4 & 2.4 & 2.4 & 2.4 \\
geometr. acc. & $\mathbf{C}$ & 2.9 & 2.9 & 2.9 & 2.9 \\
Monte Carlo corr. & $\mathbf{C}$ & 3.7 & 3.7 & 3.7 & 3.7 \\
mom. resolution & $\mathbf{B}$ & - & $<0.5$ & 0.6 & 1.2 \\
mom. scale & $\mathbf{B}$ & $<3.2$ & 3.2 & 3.5 & 3.7 \\
backgd. subtrac. & $\mathbf{B}$ & $<0.5$ & 0.6 & 3.8 & 8.2 \\
$\pi$ oversubtrac. & $\mathbf{B}$ & - & - & 1.8 & 4.7 \\
\hline Total error & & 6.2 & 6.2 & 7.6 & 11.5 \\
\hline \hline
\end{tabular}

TABLE III: Systematic errors on the $p+p$ reference spectrum.

\begin{tabular}{cccccc}
\hline \hline & type & $p_{T}<2$ & $p_{T}=4$ & $p_{T}=6$ & $p_{T}=10$ \\
\hline normalization & $\mathbf{C}$ & 10 & 10 & 10 & 10 \\
residual syst. & $\mathbf{B}$ & 3 & 5 & 7 & 9 \\
charged $^{0}\left(\pi^{0}\right)$ only $^{a}$ & $\mathbf{B}(\mathbf{C})$ & $8(5)$ & $7(5)$ & $9(5)$ & $20(5)$ \\
\hline \hline
\end{tabular}

${ }^{a}$ Errors affect only the charged (neutral) analysis, and include $\mathrm{h} / \pi_{0}$, and fits to reference (point by point $\mathrm{pp}$ ) errors.

dependent of $p_{T}$. Particle momenta are measured with a resolution $\delta p / p=0.7 \% \oplus 1.1 \% p(\mathrm{GeV} / c)$. The momentum scale is known to $0.7 \%$, from the reconstructed proton mass using the time-of-flight. A confirmation hit is required in $\mathrm{PC} 3$, located at a radius of $5 \mathrm{~m}$, within a $2.5 \sigma$ matching window to eliminate most albedo, conversions, and decays. The remaining background above $p_{T}=5 \mathrm{GeV} / c$ is subtracted statistically using identified conversions and weak decays (primarily kaons) 18].

Corrections to the charged particle spectrum for geometrical acceptance, decays in flight, reconstruction efficiency, and momentum resolution are determined using a single-particle GEANT Monte Carlo simulation. All analysis steps, including the outer detector matching cuts are applied consistently in simulation and data. As the DC and PC occupancies in $\mathrm{d}+\mathrm{Au}$ are low, no multiplicity-dependent occupancy corrections are required. The yield was corrected to the center of the $p_{T}$ bin. Table II lists the source and magnitude of each contribution to the systematic uncertainties on the charged particle spectra.

The fully corrected $p_{T}$ distributions of $\pi^{0}$ 's and $\left(h^{+}+\right.$ $\left.h^{-}\right) / 2$ are shown in Fig. 1 Each panel shows the reference spectrum from $\mathrm{p}+\mathrm{p}$ collisions along with the particle spectrum from $\mathrm{d}+\mathrm{Au}$. The reference for $\pi^{0}$ is the $\pi^{0}$ spectrum measured in $\mathrm{p}+\mathrm{p}$ by PHENIX 17], shown on the right panel. For charged hadrons, the reference is obtained by scaling up the $\pi^{0}$ spectrum by the $p_{T^{-}}$ dependent $\mathrm{h} / \pi$ ratio observed at the ISR 22] at $\sqrt{s}=23$ to $63 \mathrm{GeV}$ and measured by PHENIX. The value of $\mathrm{h} / \pi$

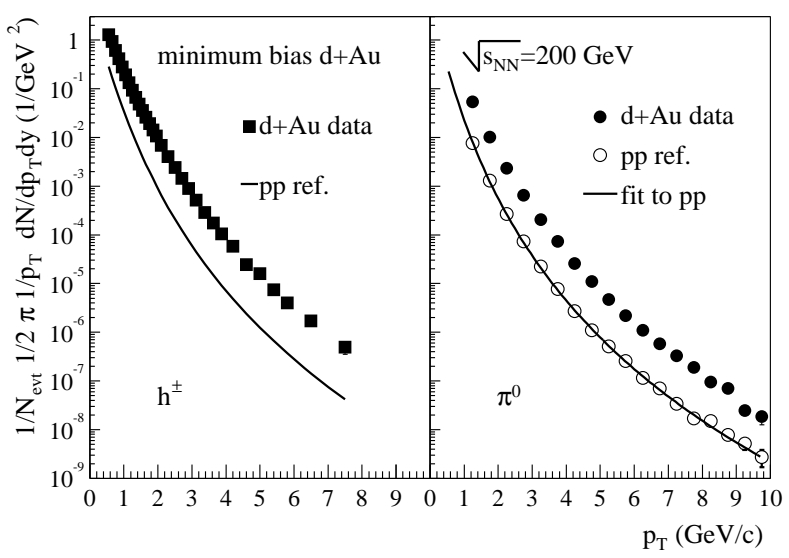

FIG. 1: Midrapidity $p_{T}$ spectra for charged hadrons and $\pi^{0}$. Total uncertainties are shown. The $\pi^{0}$ below $5 \mathrm{GeV} / c$ are from minimum bias triggered events, and above from photon triggered. Lines show fits to reference spectra from $\mathrm{p}+\mathrm{p}$ collisions, and open points show the $\mathrm{p}+\mathrm{p} \pi^{0}$ spectrum measured by PHENIX [17].

is constant at $1.6 \pm 0.16$ above $1.5 \mathrm{GeV} / c$, and decreases at lower $p_{T}$ to a value of $1.25_{-0.25}^{+0.12}$. Table III summarizes uncertainties on the reference spectra.

A standard way to quantify nuclear medium effects on high $p_{T}$ production is by the nuclear modification factor, which we define for $\mathrm{d}+\mathrm{Au}$ collisions as the ratio of invariant yield in $\mathrm{d}+\mathrm{Au}$ to that of $\mathrm{p}+\mathrm{p}$, scaled by the number of binary collisions.

$$
R_{\mathrm{dA}}\left(p_{T}\right)=\frac{\left(1 / N_{\mathrm{dA}}^{e v t}\right) d^{2} N_{\mathrm{dA}} / d \eta d p_{T}}{\left\langle N_{\text {coll }}\right\rangle / \sigma_{\mathrm{pp}}^{\text {inel }} d^{2} \sigma_{\mathrm{pp}} / d \eta d p_{T}}
$$

where $\left\langle N_{\text {coll }}\right\rangle$ is the average number of inelastic nucleonnucleon (NN) collisions per event in the minimum bias collisions, and $\left\langle N_{\text {coll }}\right\rangle / \sigma_{\mathrm{pp}}^{\text {inel }}$ is the nuclear overlap function $\left\langle T_{A}(b)\right\rangle$. Using a Glauber model [23] and simulation of the BBC, $\left\langle N_{\text {coll }}\right\rangle$ is $8.5 \pm 0.4$ in minimum bias $\mathrm{d}+\mathrm{Au}$.

The ratio $R_{\mathrm{dA}}$ is plotted separately for $\pi^{0}$ measured in the $\mathrm{PbGl}$ and in the PbSc calorimeters in Fig. 2. The two analyses are consistent within errors. The data are compared to the corresponding nuclear modification factor $R_{\mathrm{AA}}$ obtained from central $\mathrm{Au}+\mathrm{Au}$ collisions. The top panel of Fig. B shows $R_{\mathrm{dA}}$ for inclusive charged particles $\left(h^{+}+h^{-}\right) / 2$, again compared with $R_{\mathrm{AA}}$ observed in central $\mathrm{Au}+\mathrm{Au}$ collisions, while the lower panel compares $\left(h^{+}+h^{-}\right) / 2$ with $\pi^{0}$. In both Figs. 2 and 3 the uncertainties are plotted as follows: error bars represent the quadrature sum of statistical errors and those systematic errors which vary point-to-point in $p_{T}$; systematic errors on the absolute yield and the systematic errors which are correlated point-to-point are shown as bands. All procedures for estimating the various systematic errors have been adjusted to provide estimates corresponding to $1 \sigma$ error values. 


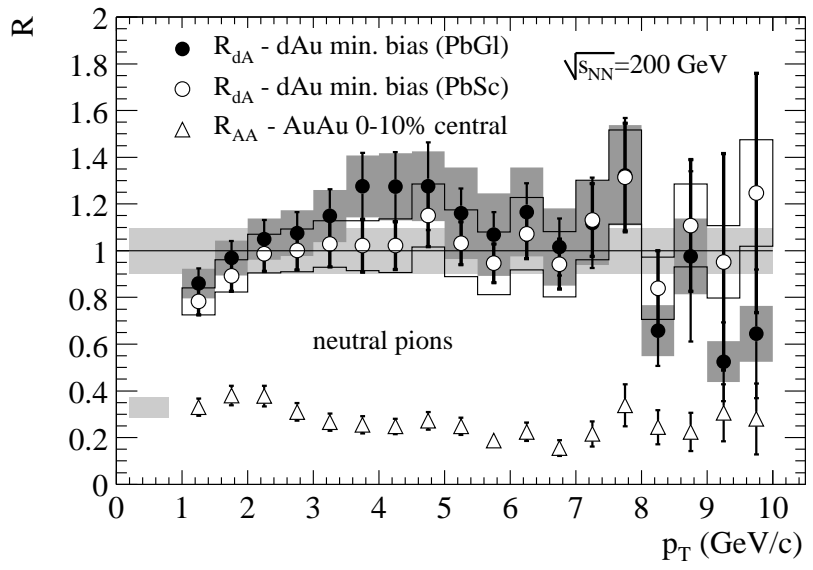

FIG. 2: Nuclear modification factor $R_{\mathrm{dA}}$ for $\pi^{0}$ in the PbGl and PbSc calorimeters in minimum bias $\mathrm{d}+\mathrm{Au}$. The bands around the data points show systematic errors which can vary with $p_{T}$, while the shaded band around unity indicates the normalization uncertainty. The nuclear modification factor $R_{\mathrm{AA}}$ in $10 \%$ most central $\mathrm{Au}+\mathrm{Au}$ collisions is also shown.

The data clearly indicate that there is no suppression of high $p_{T}$ particles in $\mathrm{d}+\mathrm{Au}$ collisions. We do, however, observe an enhancement in inclusive charged particle production at $p_{T}>2 \mathrm{GeV} / c$. A similar enhancement was observed in $\mathrm{p}+\mathrm{A}$ fixed-target experiments 24] and is generally referred to as the "Cronin effect". To facilitate comparison of the Cronin effect in inclusive charged particles and $\pi^{0}$, the lower part of Fig. B shows all systematic uncertainties common to both analyses in the bar on the left. It should be noted that this uncertainty must be added in quadrature with the bands shown for each curve to obtain the $1 \sigma$ allowed range of $R_{\mathrm{dA}}$ from the data. The $\pi^{0}$ data suggest a smaller enhancement for pions than for inclusive charged particles at $p_{T}=2$ $4 \mathrm{GeV} / c$. We note that the charged spectrum includes baryons and antibaryons, which may have a different nuclear enhancement than the mesons [24].

The various models of the suppression observed in $\mathrm{Au}+\mathrm{Au}$ predict a different dependence on $N_{\text {coll }}$ in $\mathrm{d}+\mathrm{Au}[14,[25,26]$. Therefore, a second data sample was selected by requiring observation of a neutron in the Zero-Degree Calorimeter on the deuteron-going side of PHENIX. This, together with the requirement of particles entering both Beam-Beam Counters, selects a class of events in which only the proton from the deuteron interacts with the Au nucleus. The mean number of binary collisions for this sample is calculated with the Glauber model to be $3.6 \pm 0.3$. Particle yields in this sample have a $<5 \%$ uncertainty beyond that of the minimum bias sample, arising from trigger bias.

Fig. 4 shows the ratios of $R_{\mathrm{dA}}$ in minimum bias $\mathrm{d}+\mathrm{Au}$ to $R_{\mathrm{pA}}$ in the neutron tagged sample, for both $\left(h^{+}+\right.$ $\left.h^{-}\right) / 2$ and $\pi^{0}$. Systematic uncertainties on the spectra cancel in the ratio; the band around unity shows the

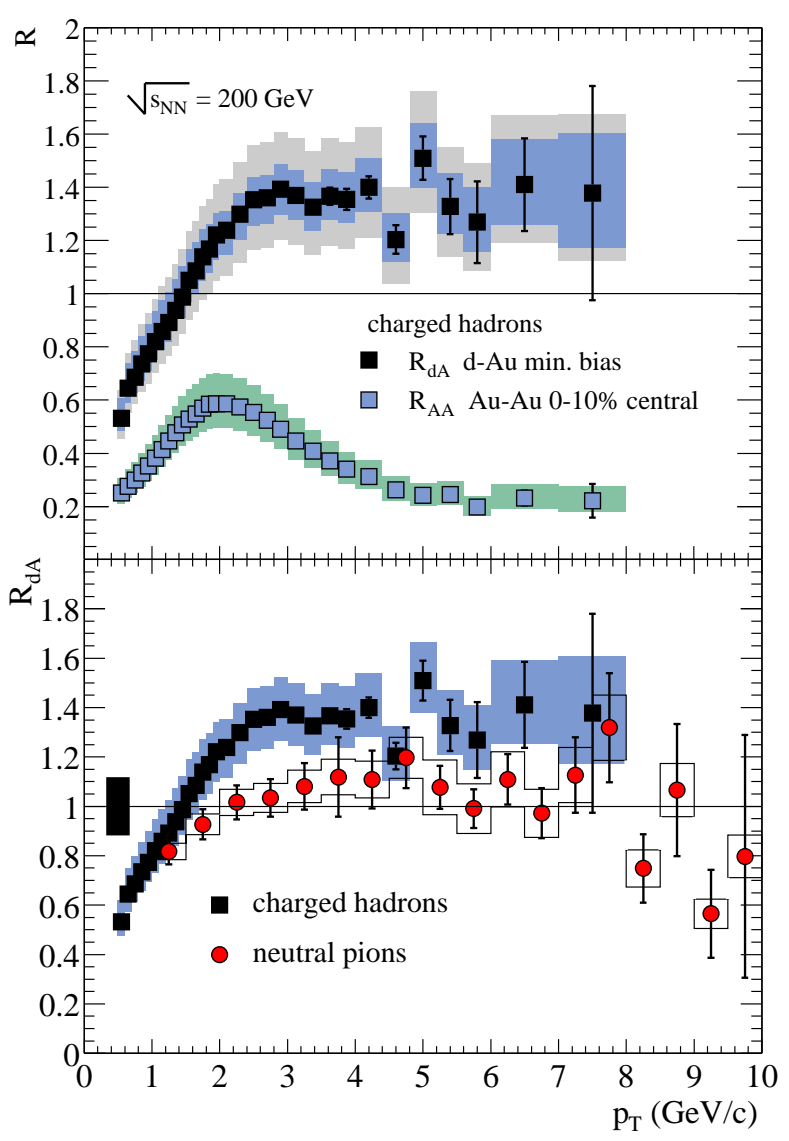

FIG. 3: Top: Nuclear modification factor $R_{\mathrm{dA}}$ for $\left(h^{+}+h^{-}\right) / 2$ in minimum bias $\mathrm{d}+\mathrm{Au}$ compared to $R_{\mathrm{AA}}$ in the $10 \%$ most central $\mathrm{Au}+\mathrm{Au}$ collisions. Inner bands show systematic errors which can vary with $p_{T}$, and outer bands include also the normalization uncertainty. Bottom: Comparison of $R_{\mathrm{dA}}$ for $\left(h^{+}+h^{-}\right) / 2$ and the average of the $\pi^{0}$ measurements in $\mathrm{d}+\mathrm{Au}$. The bar at the left indicates the systematic uncertainty in common for the charged and $\pi^{0}$ measurements.

uncertainty on the ratio of the number of binary collisions in the two samples. Average values of $N_{\text {coll }}$ are 3.6 per participating proton in the neutron tagged sample and 8.5 for 1.7 participating nucleons from the deuteron in minimum bias $\mathrm{d}+\mathrm{Au}$. Given the systematic uncertainties on $N_{\text {coll }}$, we cannot exclude a small centrality dependence for $p_{T}>1 \mathrm{GeV} / c$. It should be noted that the figure also indicates that $\mathrm{d}+\mathrm{Au}$ collisions provide a good measure of the physics of $\mathrm{p}+\mathrm{Au}$.

The observation of an enhancement of high- $p_{T}$ hadron production in both the minimum bias $\mathrm{d}+\mathrm{Au}$ and the neutron tagged sample of $\mathrm{p}+\mathrm{Au}$ collisions indicates that the suppression in central $\mathrm{Au}+\mathrm{Au}$ collisions is not an initial state effect. Nor does it arise from modification of parton structure functions in nuclei. The data suggest, instead, that the suppression of high $p_{T}$ hadrons in $\mathrm{Au}+\mathrm{Au}$ is more likely a final state effect of the produced dense medium. 


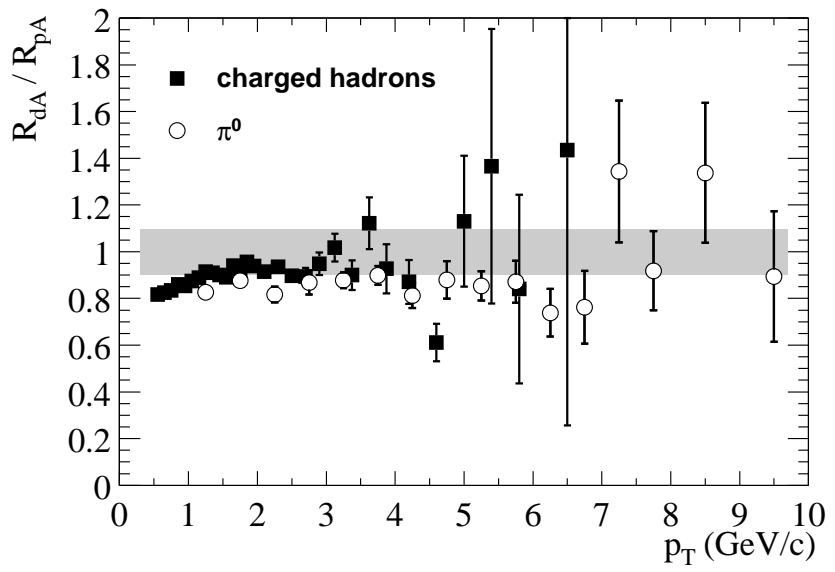

FIG. 4: Ratio of $R_{\mathrm{dA}}$ in minimum bias $\mathrm{d}+\mathrm{Au}$ and $R_{\mathrm{pA}}$ from neutron tagged $\mathrm{d}+\mathrm{Au}$ collisions for $\left(h^{+}+h^{-}\right) / 2$ and $\pi^{0}$. The band shows the uncertainty on the number of binary collisions; all other systematic errors cancel.

We thank the staff of the Collider-Accelerator and Physics Departments at BNL for their vital contributions. We acknowledge support from the Department of Energy and NSF (U.S.A.), MEXT and JSPS (Japan), CNPq and FAPESP (Brazil), NSFC (China), IN2P3/CNRS and CEA (France), BMBF, DAAD, and AvH (Germany), OTKA (Hungary), DAE and DST (India), ISF (Israel), KRF and CHEP (Korea), RMIST, RAS, and RMAE (Russia), VR and KAW (Sweden), U.S. CRDF for the FSU, US-Hungarian NSF-OTKA-MTA, and US-Israel BSF.

* PHENIX Spokesperson zajc@nevis.columbia.edu

[1] M. Gyulassy and M. Plümer, Phys. Lett. B243, 432 (1990); X.N. Wang and M. Gyulassy, Phys. Rev. Lett. 68, 1480 (1992).
[2] R. Baier et al., Phys. Lett. B345, 277 (1995).

[3] J.F. Owens et al., Phys. Rev. D18, 1501 (1978).

[4] K. Adcox et al., Phys. Rev. Lett. 88, 022301 (2002).

[5] K. Adcox et al., Phys. Lett. B561, 82 (2003).

[6] C. Adler et al., Phys. Rev. Lett. 89, 202301 (2002).

[7] S.S. Adler et al., nucl-ex/0304022 sub. to Phys. Rev. Lett.

[8] M. Gyulassy and X.N. Wang, Nucl. Phys. B420, 583 (1994); X.N. Wang, Phys. Rev. C58, 2321 (1998).

[9] I. Vitev and M. Gyulassy, Phys. Rev. Lett. 89, 252301 (2002).

[10] S. Jeon, J. Jalilian-Marian and I. Sarcevic, Phys. Lett. B562, 45 (2003).

[11] G.G. Barnaföldi et al., nucl-th/0212111. V. Greco, C.M. Ko and P. Lévai, Phys. Rev. Lett. 90, 202302 (2003).

[12] X. N. Wang, hep-ph/0301196

[13] F. Arleo, J. High Energy Phys. 11, 44 (2002).

[14] D. Kharzeev, E. Levin and L. McLerran, Phys. Lett. B561, 93 (2003).

[15] K. Gallmeister, C. Greiner and Z. Xu, Phys. Rev. C67, 044905 (2003).

[16] K. Adcox et al., Nucl. Instrum. Methods A499, 469 (2003), and references therein.

[17] S.S. Adler et al., hep-ex/0304038 submitted to Phys. Rev. Lett.

[18] J. Jia et al., Nucl. Phys. A715, 769c (2003).

[19] J. Adams et al., nucl-ex/0306024 sub. to Phys. Rev. Lett.

[20] B.B. Back et al., nucl-ex/0306025 sub. to Phys. Rev. Lett.

[21] J. T. Mitchell et al., Nucl. Instrum. Methods A482, 491 (2002).

[22] B. Alper et al., Nucl. Phys. B100, 237 (1975).

[23] In the present work the Woods-Saxon nuclear density parameters radius $R=6.38 \mathrm{fm}$, diffusivity $a=0.54 \mathrm{fm}$ and $\mathrm{N}-\mathrm{N}$ cross section $\sigma_{N N}^{i n e l}=42 \mathrm{mb}$ were used. The deuteron is described by a Hulthén wave function (L. Hulthén and M.Sagawara, Handbuch der Physik 39 (1957).) with $\alpha=$ $0.228 \mathrm{fm}^{-1}$ and $\beta=1.18 \mathrm{fm}^{-1}$.

[24] D. Antreasyan et al., Phys. Rev. D19, 764 (1979).

[25] I. Vitev, Phys. Lett. B562, 36 (2003).

[26] B. Kopeliovich, Phys. Rev. Lett. 88, 232303 (2002). 